\title{
Apigenin protects against bleomycin-induced lung fibrosis in rats
}

\author{
LING CHEN $^{1}$ and WEI ZHAO ${ }^{2}$ \\ ${ }^{1}$ Department of Respiratory Medicine, Pingmei Shenma Medical Group General Hospital, Pingdingshan, Henan 467000; \\ ${ }^{2}$ Department of Respiratory Medicine, The First Affiliated Hospital of Zhengzhou University, \\ Zhengzhou, Henan 450052, P.R. China
}

Received October 12,2014; Accepted August 26, 2015

DOI: $10.3892 /$ etm.2015.2885

\begin{abstract}
Apigenin is a non-toxic and non-mutagenic flavone that exists abundantly in numerous herbs and vegetables. Apigenin exerts anti-proliferative and anti-inflammatory properties. The aim of the present study was to investigate the effects of apigenin on bleomycin-induced lung fibrosis in rats. A single intratracheal instillation of bleomycin $(5 \mathrm{mg} / \mathrm{kg})$ was administered and rats were sacrificed on 7 and 28 days post bleomycin instillation. The instillation of bleomycin resulted in decreased body weight and an increase in the lung index. In addition, bleomycin administration increased the hydroxyproline content, myeloperoxidase (MPO) activity, tumor necrosis factor (TNF)- $\alpha$ and transforming growth factor (TGF)- $\beta$ levels and decreased the superoxide dismutase (SOD) activity in the rat lung tissues. Excessive collagen deposits were detected in the lung tissues in bleomycin-treated rats compared with normal control rats. Notably, the oral administration of apigenin $(10,15$ and $20 \mathrm{mg} / \mathrm{kg} / \mathrm{day})$ appeared to prevent the fibrotic process. The treatment suppressed the increases in hydroxyproline content, MPO activity, TNF- $\alpha$ and TGF- $\beta$ levels and attenuated the reduction of SOD activity that were induced by bleomycin. Furthermore, excessive collagen deposition was inhibited by the apigenin treatment. Collectively, these results suggest that apigenin may function as a potent anti-inflammatory and anti-fibrotic agent against bleomycin-induced lung fibrosis.
\end{abstract}

\section{Introduction}

Idiopathic pulmonary fibrosis (IPF) is a fatal interstitial lung disease characterized by rapidly progressive scarring of the lung tissue, exacerbated fibroblast proliferation, excessive deposition of matrix proteins and destruction of alveolar

Correspondence to: Dr Ling Chen, Department of Respiratory Medicine, Pingmei Shenma Medical Group General Hospital, 1 Kuanggong Road, Pingdingshan, Henan 467000, P.R. China E-mail: chenling98866@163.com

Key words: apigenin, bleomycin, lung fibrosis, tumor necrosis factor- $\alpha$, transforming growth factor- $\beta$ architecture $(1,2)$, which may result in loss of lung function and ultimately respiratory failure. The exact etiology of IPF remains unclear. Current treatments for IPF primarily include anti-inflammatory, immunosuppressive or anti-fibrotic methods, which exhibit a limited capacity to prevent the progression of the disease or to improve patient quality of life (3). In order to obtain optimal therapeutic results there is a requirement for novel therapeutic methods for the treatment of IPF.

Flavonoids are diphenyl propanoids present in edible plants and are categorized as flavonols, flavones, catechins, flavanones, anthocyanidins and isoflavonoids. Flavonoids exert anti-proliferative and anti-inflammatory properties (4-6). Apigenin (4,5,7-trihydroxyflavone) is a non-toxic and non-mutagenic flavone that exists abundantly in numerous herbs and vegetables, including chamomile, thyme, parsley and broccoli, and displays cytostatic and cytoprotective activity (7-9). However, at present it remains unclear how apigenin affects the lung fibrosis process, although it harbors anti-proliferative and anti-inflammatory properties. The aim of the present study was to investigate the potential of apigenin to treat lung fibrosis using a bleomycin-induced rat lung fibrosis model.

\section{Materials and methods}

Animals. A total of 80 male Wistar rats (age, 8 weeks; weight, 200-240 g) were obtained from Vital River Laboratories (Beijing, China). The animals were fed a standard rat chow and water ad libitum and housed in a temperature-controlled environment $\left(20-22^{\circ} \mathrm{C}\right)$ with an alternating cycle of 12-h light and dark. All rats were allowed to acclimatize for one week prior to the initiation of any experiments. The experimental design and procedures were approved by the Ethical Committee for Animal Care and Use at Pingmei Shenma Medical Group General Hospital (Pingdingshan, China).

Experimental model of bleomycin-induced lung fibrosis. Rats were randomized into 5 groups, each containing 16 animals. Group 1 consisted of saline-treated control rats and group 2 comprised bleomycin-treated rats. Animals in groups 3, 4 and 5 were treated with 10,15 and $20 \mathrm{mg} / \mathrm{kg} /$ day apigenin, respectively, starting concurrently with the induction of lung fibrosis by the administration of bleomycin and continued until the end of the experiment. Single doses of bleomycin 
(5.0 mg/kg in $1.0 \mathrm{ml}$ phosphate-buffered saline; Tianjin Taihe Pharmaceutical Co., Ltd., Tianjin, China) were injected into the rat lung intratracheally. Control animals received an identical volume of intratracheal saline instead of bleomycin. Apigenin (dissolved in olive oil; Sigma-Aldrich, St. Louis, MO, USA) was administered orally via a feeding tube. The day of bleomycin injection was defined as day 0 and the weight of the animals was recorded every 3-4 days.

Lung preparation and biochemical assays. Following the treatment, on days 7 and 28, the rats were anesthetized using $3 \%$ pentobarbital sodium and sacrificed by exsanguination at the cephalic artery. The lungs were removed and weighed and washed twice with cold saline. Then each lung was divided into two sections: The right section was fixed in $10 \%$ formalin solution for histological examination and the left section was prepared for biochemical assay and cytokine detection as described below.

The lung samples were prepared as $10 \%$ homogenates in $0.9 \%$ saline by homogenizer on ice according to their respective weight. The resulting homogenate was centrifuged, and the supernatant was collected and stored at $-20^{\circ} \mathrm{C}$ until assayed. The assays of superoxide dismutase (SOD; A001-3), myeloperoxidase (MPO; A044) and hydroxyproline (A030-2) levels were all purchased from Nanjing Jiancheng Bioengineering Institute (Nanjing, China) and performed according to the manufacturer's instructions.

Histological examination of fibrosis. Lung specimens were fixed in $10 \%$ formalin solution for $24 \mathrm{~h}$, dehydrated in ethyl alcohol and embedded in paraffin. Subsequently, 5- $\mu \mathrm{m}$ sections were stained with hematoxylin and eosin (H\&E) and Masson trichrome for histological evaluation of lung injury and fibrosis using an Olympus CKX41 light microscope (Olympus Corporation, Tokyo, Japan).

Measurement of tumor necrosis factor (TNF)- $\alpha$ and transforming growth factor (TGF)- $\beta$. Lung homogenate (10\%) was centrifuged at $12,000 \mathrm{x} \mathrm{g}$ for $30 \mathrm{~min}$ at $4^{\circ} \mathrm{C}$ to remove cell debris, and the supernatants were used for TNF- $\alpha$ and TGF- $\beta$ determination. The levels of TNF- $\alpha$ were determined using an ELISA kit from Assaypro, LLC (St. Charles, MO, USA). TGF- $\beta$ levels were assayed using a commercially available TGF- $\beta$ ELISA kit (TGF- $\beta$ E max ImmunoAssay System; Promega Corporation, Madison, WI, USA).

Statistical analysis. Statistical analysis was performed using SPSS software, version 19.0 (IBM SPSS, Armonk, NY, USA). Data are presented as the mean \pm standard deviation. Statistical differences were analyzed using one-way analysis of variance, followed by post-hoc multiple comparison tests (least significant difference). $\mathrm{P}<0.05$ was considered to indicate a statistically significant difference.

\section{Results}

Differences in the body weight and lung index. By contrast to the control group, the body weight of the bleomycin-treated group decreased gradually and reached the lowest level at day 7 after bleomycin injection (Fig. 1A). Apigenin treatment provided a dose-dependent protective effect on the body weight loss caused by bleomycin (Fig. 1A).

Lung index [weight of wet lung (mg)/body weight] is a parameter used for evaluating lung edema. In contrast with body weight loss, the lung index of the bleomycin-treated rats increased due to lung weight gain at days 7 and 28 after bleomycin injection compared with that of the normal control rats (Fig. 1B). Apigenin treatment inhibited the increase of the lung index of the bleomycin-treated rats in a concentration-dependent manner (Fig. 1B).

Effect of apigenin on hydroxyproline content, SOD activity and MPO activity. In order to assess the extent of fibrosis, the lung hydroxyproline levels were determined. As shown in Fig. 2A, at day 28 after bleomycin the lung hydroxyproline level in the bleomycin-treated rats was increased markedly compared with that of the normal control rats. Administration of apigenin reduced the hydroxyproline level in the lung homogenates in a dose-dependent manner (Fig. 2A).

The depletion of SOD indirectly indicates the production of oxygen free radicals following bleomycin injection. As shown in Fig. 2B, administration of bleomycin significantly decreased the SOD activity in the lung homogenates at day 28 after bleomycin injection. Treatment with apigenin concentration-dependently inhibited the decline of SOD activity caused by bleomycin administration (Fig. 2B).

MPO activity has been used as an index of leukocyte adhesion and accumulation in a range of tissues (10). Treatment with bleomycin elicited a profound increase in MPO activity in the lung homogenates at days 7 and 28 after bleomycin injection compared with that in the control group (Fig. 2C). Administration of apigenin led to a significant reduction in MPO activity, in a dose-dependent manner (Fig. 2C).

Effect of apigenin on pro-inflammatory and pro-fibrotic cytokine production in lung homogenates. In order to identify the function of cytokines in lung fibrosis, TNF- $\alpha$ and TGF- $\beta$ levels in lung homogenates were determined. As shown in Fig. 3A, at day 7 after bleomycin administration the level of TNF- $\alpha$ increased markedly compared with those in normal control rats. Apigenin suppressed the increase of TNF- $\alpha$ level dose-dependently (Fig. 3A). In addition, treatment of rats with bleomycin elicited a notable increase in TGF- $\beta$ levels in the lung homogenates at days 7 and 28 after bleomycin injection compared with that in normal control rats (Fig. 3B). Administration of apigenin inhibited the increase of TGF- $\beta$ level caused by bleomycin treatment in a concentration-dependent manner (Fig. 3B).

Histological differences. Rat lungs were examined histologically on day 7 after bleomycin injection (Fig. 4). The lung architecture in the control group appeared unaffected (Fig. 4A), while the bleomycin-treated rats exhibited collapsed and narrowed alveoli, marked thickening of the interalveolar septa and dense interstitial infiltration by inflammatory cells (Fig. 4B). In addition, Masson trichrome staining results demonstrated that there was excessive collagen deposition in the lung tissues of the bleomycin-treated rats (Fig. 4E) compared with the control rats (Fig. 4D). In contrast to the bleomycin-treated rats (Fig. 4B), the rats treated with 

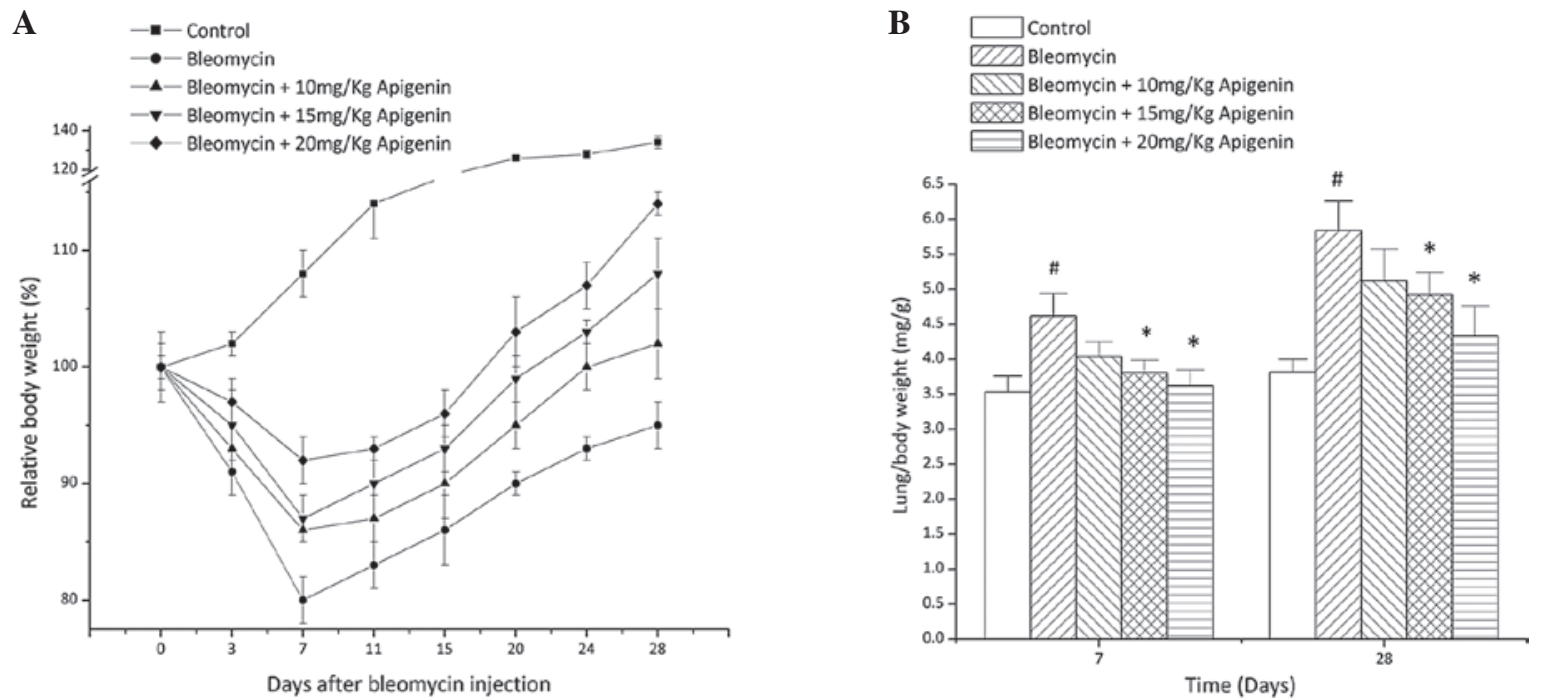

Figure 1. (A) Apigenin inhibited body weight loss following bleomycin treatment. Rats were randomized into weight-matched groups. The body weight on day 0 was defined as $100 \%$. The relative body weight was calculated as a percentage of that measured on day 0. (B) Apigenin decreased lung index in the bleomycin-treated rats. Rats were sacrificed on days 7 and 28 after bleomycin injection. Lung index was calculated as the ratio of lung wight (mg) to body weight $(\mathrm{g})$ of each rat. Values are expressed as the mean \pm standard deviation $(\mathrm{n}=8)$. Experiments were repeated three times independently, and produced similar results. ${ }^{~} \mathrm{P}<0.05$ vs. control group; ${ }^{*} \mathrm{P}<0.05$ vs. bleomycin group.
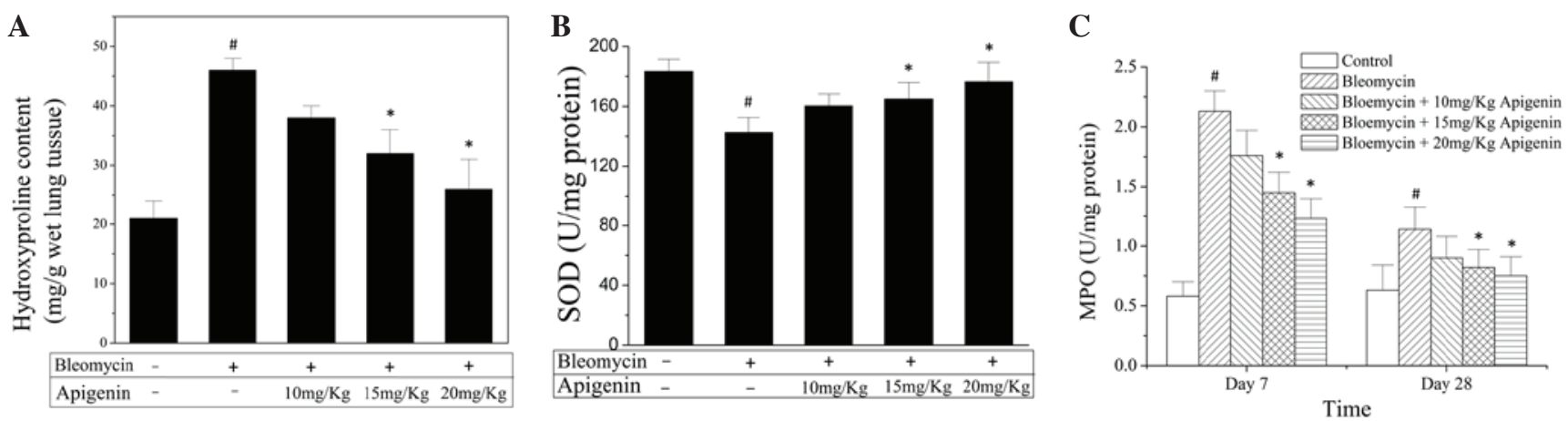

Figure 2. Influence of apigenin on the hydroxyproline content, SOD and MPO activity in the rat lung tissues. (A) Hydroxyproline content and (B) SOD activity were measured on day 28 after bleomycin injection; the rats were sacrificed and the hydroxylproline content or SOD activity was measured. (C) MPO activity. On days 7 and 28 after bleomycin injection rats were sacrificed and the MPO activity was determined using a commercially available kit. Data are presented as the mean \pm standard deviation $(n=8)$. Experiments were repeated three times independently, and produced similar results. ${ }^{~} \mathrm{P}<0.05$ vs. control group; ${ }^{*} \mathrm{P}<0.05$ vs. bleomycin group. SOD, superoxide dismutase; MPO, myeloperoxidase.

bleomycin and $20 \mathrm{mg} / \mathrm{kg}$ apigenin presented with a significant suppression of inflammatory cell infiltration, reduced thickening of the interalveolar septa and increased inflation of the alveoli (Fig. 4C). Furthermore, $20 \mathrm{mg} / \mathrm{kg}$ apigenin treatment markedly reduced collagen deposition in the lung tissues of the bleomycin-treated rats, as indicated by Masson trichrome staining (Fig. 4F).

\section{Discussion}

Previous studies have demonstrated the anti-inflammatory activity of apigenin under a range of pathophysiological conditions. Apigenin has been shown to block lipopolysaccharide (LPS)-induced lethality in vivo and the expression of proinflammatory cytokines by inactivating NF- $\kappa B$ via the suppression of $\mathrm{p} 65$ phosphorylation (10). In endothelial cells, apigenin provides a protective effect against LPS-induced inflammation by decreasing caspase- 3 activation and modulating mitochondrial function (11). Apigenin exerts anti-inflammatory activity via multiple mechanisms (12), including the induction of apoptosis and the regulation of MAPK signaling pathways in mouse macrophage ANA-1 cells (13). Other mechanisms include the inhibition of inflammatory cytokine production. Apigenin has demonstrated the ability to suppress the expression of interleukin (IL)- $1 \beta$ and tumor necrosis factor (TNF)- $\alpha$ at the genetic level in J774.2 macrophages (14). Apigenin has also been shown to inhibit the expression of IL-6, IL-8 and intracellular adhesion molecule 1 in bis(2-ethylhexyl)phthalate-stimulated human umbilical vein endothelial cells and in vivo (15). In cystic fibrosis bronchial cells, the expression of the pro-inflammatory IL-8 gene was suppressed by apigenin (16). In addition, apigenin has been indicated to exert a protective effect against oxidative stress (17-19). Furthermore, apigenin inhibits TGF- $\beta 1$-induced collagen production and fibroblast-to-myofibroblast transition in human lung fibroblast populations $(20,21)$. Although apigenin exhibits numerous activities that are potentially preventative of fibrotic disease, 
A

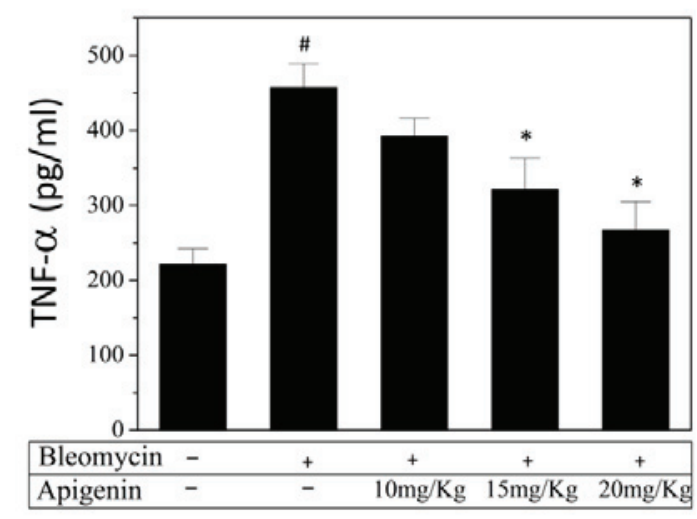

B

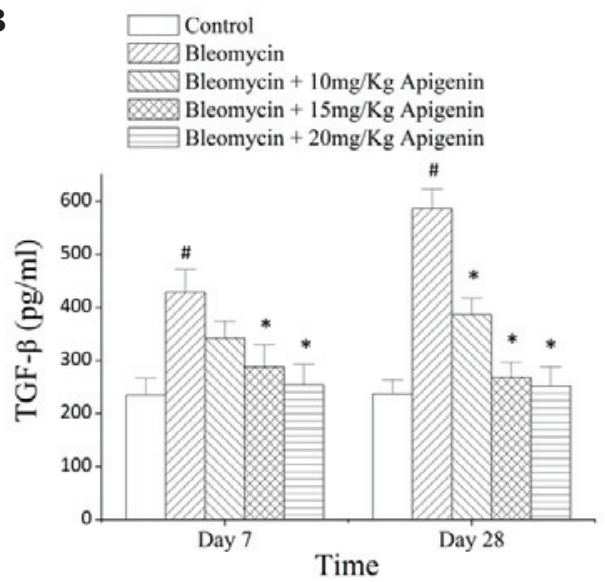

Figure 3. Apigenin inhibited TNF- $\alpha$ and TGF- $\beta$ production in the lung homogenates of bleomycin-treated rats. (A) TNF- $\alpha$; rats were sacrificed on day 7 and lungs were removed and homogenized. Supernatants were analyzed for TNF- $\alpha$ level. (B) TGF- $\beta$; rats were sacrificed on days 7 and 28 after bleomycin injection. Lungs were removed and homogenized. Following centrifugation, the supernatants were assayed for TGF- $\beta$ levels. Values are expressed as the mean \pm standard deviation $(\mathrm{n}=8)$. Experiments were repeated three times independently, and produced similar results. "P<0.05 vs. control group; ${ }^{*} \mathrm{P}<0.05$ vs. bleomycin group. TNF- $\alpha$, tumor necrosis factor- $\alpha$; TGF- $\beta$, transforming growth factor- $\beta$.

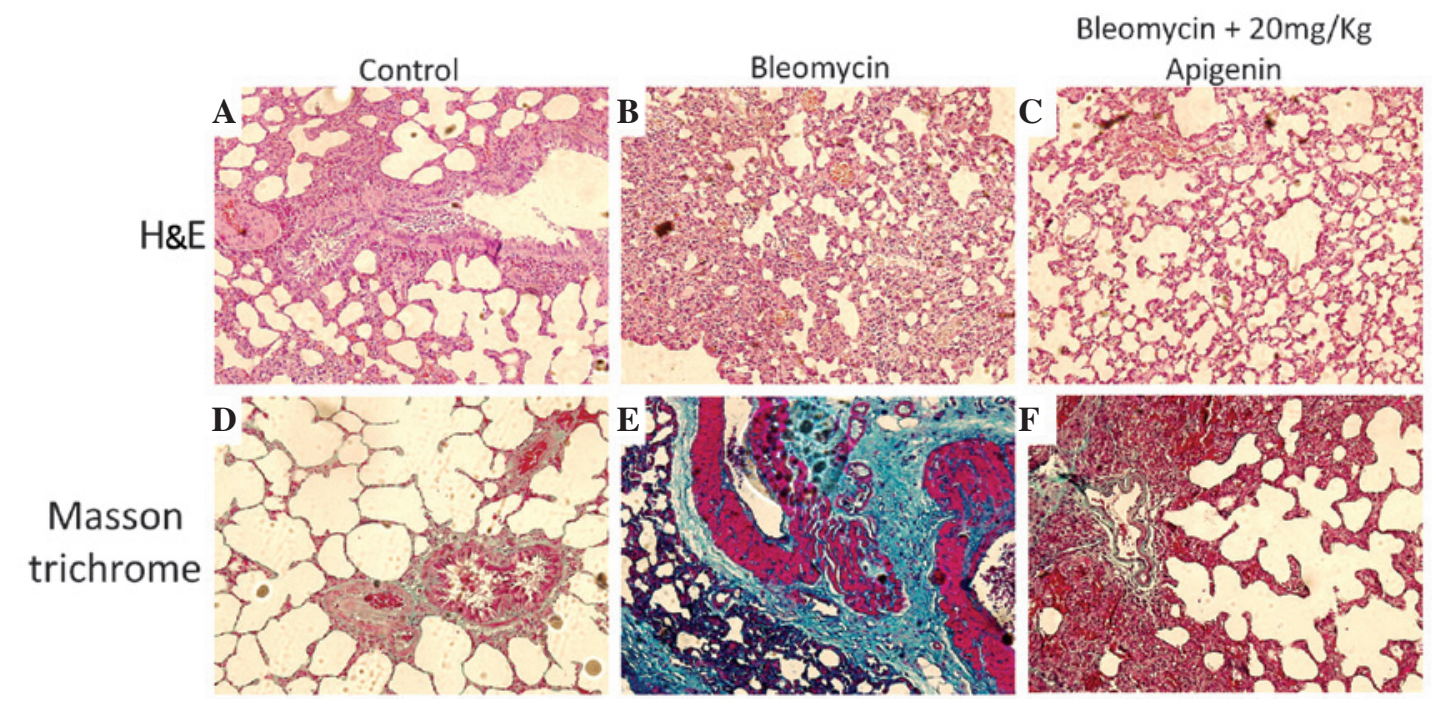

Figure 4. Photomicrographs of rat lung sections. Rats were sacrificed on day 7 after bleomycin injection. Lungs were removed, fixed in $10 \%$ formalin solution, dehydrated in ethyl alcohol and embedded in paraffin. Sections of $5 \mu \mathrm{m}$ thickness were stained with H\&E or Masson trichrome. (A-C) H\&E stained, (D-F) Masson trichrome stained. (A and D) Control group, (B and E) bleomycin group and (C and F) bleomycin $+20 \mathrm{mg} / \mathrm{kg}$ apigenin-treated group (magnification, x200). H\&E, hematoxylin and eosin.

the impact of apigenin on lung fibrosis processes previously remained unclear.

In the present study, the protective effect of apigenin on bleomycin-induced lung fibrosis was investigated in rats. Bleomycin administration resulted in body weight loss, lung index increase, progressive and significant inflammation, exacerbated fibrosis and severe alveolar destruction in rat lungs. In addition, significant reductions in SOD activity and elevation of hydroxyproline content, MPO activity, TNF- $\alpha$ and TGF- $\beta 1$ levels were detected in the rat lung tissues following bleomycin exposure. However, apigenin treatment prevented bleomycin-induced lung fibrosis and inflammation, decreased the TNF- $\alpha$ and TGF- $\beta 1$ levels and attenuated the reduction of SOD activity in rat lungs. Overall, the present results suggest that apigenin exerts a protective effect against bleomycin-induced lung fibrosis in rats.
Bleomycin-induced animal lung injury has been widely used as a model of human lung fibrosis as certain biochemical and functional changes in the early stages in animals resemble those observed in humans. The inflammatory responses observed following bleomycin administration include leukocytes infiltration. Infiltrated leukocytes are able to synthesize and secrete various cytokines, chemokines, reactive oxygen species and proteases, that may sustain injury/repair processes $(22,23)$. MPO activity has been used as an index of leukocyte infiltration. As shown in Fig. 2C, apigenin inhibited the bleomycin-induced increase of MPO activity, suggesting that apigenin inhibited leukocyte infiltration.

A number of studies have demonstrated that redox state and oxidant-antioxidant balance serve a crucial function in the pathogenesis of lung fibrosis in animal models, and potentially in humans $(24,25)$. High levels of oxidants enhance TGF- $\beta$ 
production (26), activate protease and promote the fibrotic response. Certain antioxidants, including SOD, have been observed to inhibit collagen deposition and protect the lungs in a range of animal models (27). As shown in Fig. 2B, apigenin was able to reverse the bleomycin-induced reduction of SOD activity and thus provide a protective effect.

Cytokines are involved in lung fibrosis. TNF- $\alpha$ functions as a chemokine in inflammatory cells, in addition to promoting the synthesis of fibronectin, prostaglandin and TGF- $\beta$. Depletion of TNF- $\alpha$ by antibodies has demonstrated a beneficial effect in the inhibition of bleomycin-induced lung injury $(28,29)$. In the present study, a marked increase in TNF- $\alpha$ levels in the lungs occurred following bleomycin exposure, which was suppressed by apigenin treatment in a dose-dependent manner (Fig. 3A). TGF- $\beta$ is a key cytokine associated with the induction of lung injury, and it contributes to lung fibrosis via the induction of collagen gene expression or synthesis by stimulation of fibroblast proliferation (30-33). In the present study, TGF- $\beta$ production in the lung homogenates was increased following bleomycin injection, while apigenin treatment inhibited TGF- $\beta$ production, retaining it at a basal level comparable with that detected in the normal control group (Fig. 3B). Thus, the reduced hydroxyproline production (Fig. 2A) and lung injury observed in the apigenin-treated rats may be partially attributed to the capacity of apigenin to inhibit TGF- $\beta$ production.

In summary, apigenin demonstrated protective effects against bleomycin-induced lung fibrosis in rats. The beneficial effect of apigenin may be associated with its anti-inflammatory, antioxidative and cytokine inhibition capacities.

\section{References}

1. Gross TJ and Hunninghake GW: Idiopathic pulmonary fibrosis. N Engl J Med 345: 517-525, 2001

2. Dempsey OJ and Miller D: Idiopathic pulmonary fibrosis. BMJ 347: f6579, 2013

3. Mahendran S and Sethi T: Treatments in idiopathic pulmonary fibrosis: Time for a more targeted approach? QJM 105: 929-934, 2012.

4. Villar A, Gasco MA and Alcaraz MJ: Anti-inflammatory and anti-ulcer properties of hypolaetin-8-glucoside, a novel plant flavonoid. J Pharm Pharmacol 36: 820-823, 1984.

5. Gerdin B and Svensjö E: Inhibitory effect of the flavonoid $\mathrm{O}$-(beta-hydroxyethyl)-rutoside on increased microvascular permeability induced by various agents in rat skin. Int J Microcirc Clin Exp 2: 39-46, 1983.

6. Agarwal OP: The anti-inflammatory action of nepitrin, a flavonoid. Agents Actions 12: 298-302, 1982.

7. Clere N,Faure S, Martinez MC and Andriantsitohaina R: Anticancer properties of flavonoids: Roles in various stages of carcinogenesis. Cardiovasc Hematol Agents Med Chem 9: 62-77, 2011.

8. Czyz J,Madeja Z, Irmer U, Korohoda W and Hülser DF: Flavonoid apigenin inhibits motility and invasiveness of carcinoma cells in vitro. Int J Cancer 114: 12-18, 2005.

9. Shukla S and Gupta S: Apigenin-induced cell cycle arrest is mediated by modulation of MAPK, PI3K-Akt and loss of cyclin D1 associated retinoblastoma dephosphorylation in human prostate cancer cells. Cell Cycle 6: 1102-1114, 2007.

10. Nicholas C, Batra S, Vargo MA, Voss OH, Gavrilin MA, Wewers MD, Guttridge DC, Grotewold E and Doseff AI: Apigenin blocks lipopolysaccharide-induced lethality in vivo and proinflammatory cytokines expression by inactivating NF-kappaB through the suppression of p65 phosphorylation. J Immunol 179: 7121-7127, 2007.

11. Duarte S, Arango D, Parihar A, Hamel P, Yasmeen R and Doseff AI: Apigenin protects endothelial cells from lipopolysaccharide (LPS)-induced inflammation by decreasing caspase-3 activation and modulating mitochondrial function. Int J Mol Sci 14: 17664-17679, 2013.
12. Zhang X, Wang G, Gurley EC and Zhou H: Flavonoid apigenin inhibits lipopolysaccharide-induced inflammatory response through multiple mechanisms in macrophages. PloS One 9: e107072, 2014

13. Liao Y, Shen W, Kong G, Lv H, Tao W and Bo P: Apigenin induces the apoptosis and regulates MAPK signaling pathways in mouse macrophage ANA-1 cells. PloS One 9: e92007, 2014.

14. Kowalski J, Samojedny A, Paul M, Pietsz G and Wilczok T: Effect of apigenin, kaempferol and resveratrol on the expression of interleukin-1beta and tumor necrosis factor-alpha genes in J774.2 macrophages. Pharmacol Rep 57: 390-394, 2005.

15. Wang J, Liao Y, Fan J, Ye T, Sun X and Dong S: Apigenin inhibits the expression of IL-6, IL-8 and ICAM-1 in DEHP-stimulated human umbilical vein endothelial cells and in vivo. Inflammation 35: 1466-1476, 2012.

16. Lampronti I, Borgatti M, Vertuani S, Manfredini S and Gambari R: Modulation of the expression of the proinflammatory IL-8 gene in cystic fibrosis cells by extracts deriving from olive mill waste water. Evid Based Complement Alternat Med 2013: 960603, 2013.

17. Huang CS, Lii CK, Lin AH, Yeh YW, Yao HT, Li CC, Wang TS and Chen HW: Protection by chrysin, apigenin and luteolin against oxidative stress is mediated by the Nrf2-dependent up-regulation of heme oxygenase 1 and glutamate cysteine ligase in rat primary hepatocytes. Arch Toxicol 87: 167-178, 2013

18. Han JY, Ahn SY, Kim CS, Yoo SK, Kim SK, Kim HC, Hong JT and Oh KW: Protection of apigenin against kainate-induced excitotoxicity by anti-oxidative effects. Biol Pharm Bull 35: 1440-1446, 2012.

19. Jin BH, Qian LB, Chen S, Li J, Wang HP, Bruce IC, Lin J and $\mathrm{Xia} \mathrm{Q}:$ Apigenin protects endothelium-dependent relaxation of rat aorta against oxidative stress. Eur J Pharmacol 616: 200-205, 2009.

20. Ricupero DA, Poliks CF, Rishikof DC, Kuang PP and Goldstein RH: Apigenin decreases expression of the myofibroblast phenotype. FEBS Lett 506: 15-21, 2001

21. Wojcik KA, Skoda M, Koczurkiewicz P, Sanak M, Czyż J and Michalik M: Apigenin inhibits TGF- $\beta 1$ induced fibroblast-to-myofibroblast transition in human lung fibroblast populations. Pharmacol Rep 65: 164-172, 2013.

22. Tarnell EB, Oliver BL, Johnson GM, Watts FL and Thrall RS: Superoxide anion production by rat neutrophils at various stages of bleomycin-induced lung injury. Lung 170: 41-50, 1992.

23. Oury TD, Thakker K, Menache M, Chang LY, Crapo JD and Day BJ: Attenuation of bleomycin-induced pulmonary fibrosis by a catalytic antioxidant metalloporphyrin. Am J Respir Cell Mol Biol 25: 164-169, 2001.

24. Kinnula VL, Fattman CL, Tan RJ and Oury TD: Oxidative stress in pulmonary fibrosis: A possible role for redox modulatory therapy. Am J Respir Crit Care Med 172: 417-422, 2005

25. Kuwano K, Nakashima N, Inoshima I, Hagimoto N, Fujita M, Yoshimi M, Maeyama T, Hamada N, Watanabe K and Hara N: Oxidative stress in lung epithelial cells from patients with idiopathic interstitial pneumonias. Eur Respir J 21: 232-240, 2003.

26. Bellocq A, Azoulay E, Marullo S, Flahault A, Fouqueray B, Philippe C, Cadranel J and Baud L: Reactive oxygen and nitrogen intermediates increase transforming growth factor-betal release from human epithelial alveolar cells through two different mechanisms. Am J Respir Cell Mol Biol 21: 128-136, 1999.

27. Salvemini D, Riley DP and Cuzzocrea S: SOD mimetics are coming of age. Nat Rev Drug Discov 1: 367-374, 2002.

28. Piguet PF, Ribaux C, Karpuz V, Grau GE and Kapanci Y: Expression and localization of tumor necrosis factor-alpha and its mRNA in idiopathic pulmonary fibrosis. Am J Pathol 143: 651-655, 1993.

29. Yara S, Kawakami K, Kudeken N, Tohyama M, Teruya K, Chinen T, Awaya A and Saito A: FTS reduces bleomycin-induced cytokine and chemokine production and inhibits pulmonary fibrosis in mice. Clin Exp Immunol 124: 77-85, 2001.

30. Bhatia M, Zemans RL and Jeyaseelan S: Role of chemokines in the pathogenesis of acute lung injury. Am J Respir Cell Mol Biol 46: 566-572, 2012.

31. Martin TR and Matute-Bello G: Experimental models and emerging hypotheses for acute lung injury. Crit Care Clin 27: 735-752, 2011.

32. Anscher MS: Targeting the TGF-betal pathway to prevent normal tissue injury after cancer therapy. Oncologist 15: 350-359, 2010.

33. Wilson MS, Madala SK, Ramalingam TR, Gochuico BR, Rosas IO, Cheever AW and Wynn TA: Bleomycin and IL-1beta-mediated pulmonary fibrosis is IL-17A dependent. J Exp Med 207: 535-552, 2010. 